\title{
Isospin-symmetry restoration within the nuclear density functional theory: formalism and applications
}

\author{
W. Satuła, ${ }^{1}$ J. Dobaczewski, ${ }^{1,2}$ W. Nazarewicz, ${ }^{3,4,1}$ and M. Rafalski ${ }^{1}$ \\ ${ }^{1}$ Institute of Theoretical Physics, University of Warsaw, ul. Hoża 69, 00-681 Warsaw, Poland \\ ${ }^{2}$ Department of Physics, P.O. Box 35 (YFL), FI-40014 University of Jyväskylä, Finland \\ ${ }^{3}$ Department of Physics \& Astronomy, University of Tennessee, Knoxville, Tennessee 37996 \\ ${ }^{4}$ Physics Division, Oak Ridge National Laboratory, P.O. Box 2008, Oak Ridge, Tennessee 37831
}

(Dated: October 25, 2018)

\begin{abstract}
Isospin symmetry of atomic nuclei is explicitly broken by the charge-dependent interactions, primarily the Coulomb force. Within the nuclear density functional theory, isospin is also broken spontaneously. We propose a projection scheme rooted in a mean field theory, that allows the consistent treatment of isospin breaking in both ground and exited nuclear states. We demonstrate that this scheme is essentially free from spurious divergences plaguing particle-number and angularmomentum restoration approaches. Applications of the new technique include excited high-spin states in medium-mass $N=Z$ nuclei, such as superdeformed bands and many-particle-many-hole terminating states.
\end{abstract}

PACS numbers: 21.10.Hw, 21.60.Jz, 21.30.Fe, 27.40.+z, 71.15.Mb

\section{INTRODUCTION}

Density functional theory (DFT) in its original formulation [1, 2] and its extended versions [3, 4, [5, 6] has became a universal approach to compute the ground-state (g.s.) and excited [7, 8] configurations of many-electron systems held together by an external one-body potential in condensed-matter, atomic, and molecular physics. The DFT strategy has also been used in the area of nuclear structure. The nuclear DFT, a natural extension of the self-consistent mean-field (MF) theory [9, 10] is a tool of choice for computations of g.s. properties and low-lying excitations of medium-mass and heavy nuclei.

There are a number of differences between the electronic DFT and nuclear DFT. First of all, because of the absence of external potential, nuclei are selfbound systems, and this creates conceptual problems due to the distinction between intrinsic (symmetry-broken) and laboratory-system densities. This problem can be treated within the extended DFT framework of LevyLieb [3, 4, 5, 6, 11], see Refs. 12, 13, 14, 15]. The second difference is related to the effective interaction. A complicated form of the effective nucleon-nucleon interaction, as well as complexity of the nuclear matter saturation-mechanism (pertaining to three-body forces), give rise to the presence of higher-order terms in the nuclear energy density functional (EDF). Hence, the EDF cannot be constructed in a model-independent $a b$ initio way based solely on bulk properties of infinite-medium augmented by exchange and gradient corrections, as it is usually done for electronic systems. The current best nuclear EDFs are still phenomenological in nature, either inspired by a MF averaging of the effective interaction such as the zero-range Skyrme [16] force, or based on systematic expansion involving symmetry-constrained gradient terms [17]. By construction, the nuclear EDF is characterized by a certain number of free parameters which are fitted directly to empirical data.

The third major difference between electronic and nucleonic DFT, addressed in this study, is the presence of two types of fermions and the charge independence of the nuclear interactions giving rise to the isospin symmetry. Since the isospin symmetry is violated essentially by the Coulomb interaction, which is much weaker than the strong interaction between nucleons, many effects associated with the isospin breaking in nuclei can be treated in a perturbative way, making the formalism of isotopic spin a very powerful concept in nuclear structure and reactions [18, 19].

Up to electromagnetic effects, the isospin symmetry should be conserved by elementary excitations of nuclei. This is not the case for the elementary excitations modes of the nuclear Hartree-Fock (HF) or Kohn-Sham theory, i.e., proton and/or neutron particle-hole (p-h) excitations. The independent-particle wave function manifestly breaks the isospin symmetry in the g.s. configurations of odd-odd $N=Z$ nuclei and in all other but isoscalar excited states. Two prominent examples are discussed in this work: superdeformed (SD) bands in a doubly magic nucleus ${ }^{56} \mathrm{Ni}[20]$ and terminating states in $N=Z A \sim 40$ nuclei [21].

The paper is organized as follows. We begin in Sec. II with general discussion of g.s. configurations of odd-odd $N=Z$ nuclei and p-h excitations in even-even (e-e) nuclei, so as to qualitatively introduce problems faced by MF-based theories around $N=Z$ and to provide motivation for the necessity of isospin restoration. Section [II contains a detailed presentation of the isospin-projected DFT approach. In particular, we demonstrate that this approach is essentially free from problems related to uncompensated poles plaguing projection techniques 22]; hence, it requires no further regularization 23]. Applications of the isospin-projected DFT approach to the SD bands in ${ }^{56} \mathrm{Ni}$ and terminating states in $N=Z$ isotopes of $\mathrm{Ca}, \mathrm{Sc}, \mathrm{Ti}, \mathrm{V}$, and $\mathrm{Cr}$, are presented in Secs. IVA 


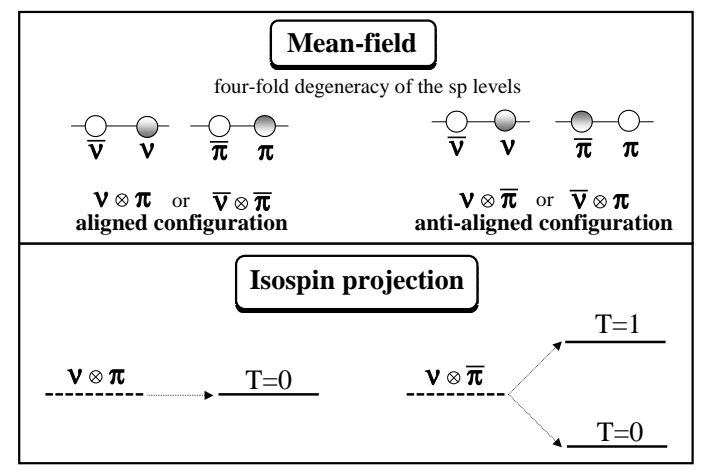

FIG. 1: The upper panel schematically shows two possible g.s. configurations of an odd-odd $N=Z$ nucleus, as described by the conventional deformed MF theory. These degenerate configurations are called aligned (left) and anti-aligned (right), depending on what levels are occupied by the valence particles. The lower panel shows what happens if the isospinsymmetry is restored. The aligned configuration is isoscalar; hence, it is insensitive to the isospin projection. The antialigned configuration represents a mixture of the $T=0$ and $T=1$ states. The isospin projection removes the degeneracy by shifting the $T=0$ component down.

and IVB, respectively. Finally, Sec. $\mathrm{V}$ contains the conclusions of this work.

\section{ISOSPIN SYMMETRY VIOLATION IN A MEAN-FIELD APPROACH}

Let us begin by exposing serious problems with the MF description of g.s. configurations of odd-odd (o-o) $N=Z$ nuclei. If, for the sake of simplicity, the Coulomb and time-odd polarization effects are disregarded and proton-neutron symmetry is conserved, a deformed MF approach naturally leads to four-fold degenerate (isospin and Kramers) single-particle (s.p.) levels. Consequently, the MF g.s. configuration of the o-o $N=Z$ nucleus is not uniquely defined and depends on occupation of specific levels. As shown in the upper panel of Fig. 1, the valence proton and neutron can be arranged in two distinctively different ways. Indeed, configurations of the type shown on the left-hand side (aligned configurations), are symmetric in spin-space coordinates and, therefore, antisymmetric (isoscalar) in isospin coordinates. The antialigned configurations depicted on the right-hand side, have a mixed symmetry in spin-space coordinates and, therefore, also in isospin coordinates.

Because of their isoscalar character, aligned configurations are not affected by the isospin projection. On the other hand, the projection lifts the degeneracy of $T=0$ and $T=1$ components of aligned configurations as it is illustrated in the lower panel of Fig. 1. Due to the repulsive character of the nuclear symmetry energy, the isovector (isoscalar) components of the anti-aligned configurations are shifted up (down) in energy. Hence, isospin restoration changes the structure of the ground states of $\mathrm{O}-\mathrm{O}$

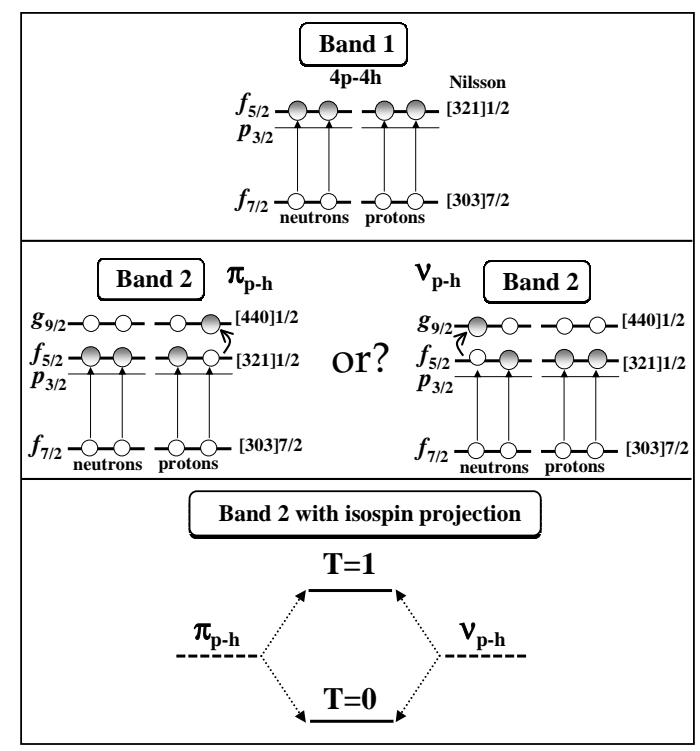

FIG. 2: The upper panel schematically shows the s.p. configuration corresponding to the SD Band 1 in ${ }^{56} \mathrm{Ni}$, which is based on a $4 \mathrm{p} 4 \mathrm{~h}$ excitation with respect to the doubly magic core. The middle panel depicts two possible configurations of Band 2 involving a single proton (left) or neutron (right) promoted to the $0 g_{9 / 2}$ shell. To facilitate the discussion, we also show the relevant asymptotic Nilsson quantum numbers associated with deformed orbitals involved. The lower panel illustrates the isospin splitting induced by the isospin-symmetry restoration.

$N=Z$ nuclei without affecting the ground states of e-e $N=Z$ nuclei. In other words, it does affect the bindingenergy staggering along the $N=Z$ line.

Another example that nicely illuminates problems with isospin encountered in MF approaches is the case of two SD bands observed in the doubly magic nucleus ${ }^{56} \mathrm{Ni}[20]$. Following Ref. [20], we label them as Band 1 and Band 2. Band 1 is interpreted as a four-particle four-hole configuration formed by promoting two protons and two neutrons from the $0 f_{7 / 2}$ shell to $0 f_{5 / 2}$ shell. Within deformed MF model, where it is more natural to use the notion of Nilsson orbitals, Band 1 is obtained by emptying the neutron and proton [303]7/2 Nilsson extruder orbitals and occupying the prolate-driving [321]1/2 levels. This interpretation, not involving the $0 g_{9 / 2}$ shell, is strongly supported by both state-of-the-art shell-model (SM) calculations [20, 24] in the $0 \hbar \omega f p$ space and the self-consistent cranked-HF theory [20]. Both approaches appear to reproduce satisfactorily the excitation energy and moment of inertia (MoI) of this band. The p-h configuration of this band is depicted schematically in the upper panel of Fig. 2 .

Band 2, on the other hand, cannot be reproduced within the $f p$ SM space. This band has also a larger MoI as compared to the Band 1 , and it is becoming yrast around spin $I \sim 12 \hbar$. These two facts strongly suggest that its structure must involve at least one particle in the prolate-driving [440]1/2 Nilsson orbital that originates 
from the $0 g_{9 / 2}$ shell. According to the calculations of Ref. [20], Band 2 involves one proton in $\pi[440] 1 / 2$ state. Its configuration, which can be regarded as one proton p-h excitation with respect to the reference Band 1, is schematically shown in Fig. 2 (middle panel, left-hand side). This scenario has been supported by the full $p f-$ and $p f g_{9 / 2}$-SM calculations [25].

The conventional MF interpretation of Band 2 is not fully supported by experiment. Indeed, the MF theory predicts existence of the second band that is built upon the single neutron occupying the $\nu[440] 1 / 2$ orbital (middle panel of Fig. 2. right-hand side). This neutron band is predicted to be placed only slightly higher in energy than the proton band, and this is due to a tiny difference in the Coulomb energies between these two configurations with, otherwise, very similar properties. Therefore, it is extremely difficult to understand within MF theory why only one of these two bands is observed experimentally. Moreover, HF calculations predict that the $\pi[440] 1 / 2$ band is too high in excitation energy and that it crosses Band 1 above spin $I \sim 16 \hbar$, that is, well above the empirical crossing. In these discussion, energetic arguments essentially exclude configurations involving two or more particles in the $0 g_{9 / 2}$ shell.

Here again the problems with the MF approach can be traced back to the isospin-symmetry violation by the $\mathrm{p}-\mathrm{h}$ excitations. Indeed, the MF approach treats proton $\left|\pi_{p-h}\right\rangle$ and neutron $\left|\nu_{p-h}\right\rangle$ p-h excitations as independent elementary excitations, and this manifestly breaks the isospin symmetry in $N=Z$ nuclei. To make the elementary excitation modes in $N=Z$ nuclei consistent with the symmetry, one needs to symmetrize or anti-symmetrize the Slater determinants corresponding to the mirror $\mathrm{p}-\mathrm{h}$ excitations $|T=0(1)\rangle \approx \frac{1}{\sqrt{2}}\left(\left|\pi_{p-h}\right\rangle \pm\left|\nu_{p-h}\right\rangle\right)$. Such wave functions go beyond the usual MF picture. Restoration of isospin lifts the MF degeneracy between proton and neutron excitations and shifts the isoscalar (isovector) configurations down (up) in energy, as illustrated in the lower panel of Fig. 2 Of course, it is irrelevant whether the $T=0$ and $T=1$ components are projected from the proton or neutron state; the results should be identical up to the tiny polarization effects.

At present, it is not at all clear how to include the isospin correlations directly into the functional. The most natural method of taking them it into account is the isospin projected DFT approach [26, 27, 28, 29], which is also used in this work. We note that such an approach is within the provisos of the DFT, whereby the isospin-projected energy is still a functional of the isospin-unprojected density, cf. Refs. 30, 31, 32].

We finish the discussion in this Section by noticing that even the isospin-projected DFT approach cannot fully account for the structure of the g.s. of an o-o $N=Z$ nucleus. Indeed, within the conventional DFT approach, the aligned and anti-aligned configurations differ due to different time-odd (TO) polarization effects in these two configurations. This polarization affects the position of the $T=1, T_{z}=0$ states with respect to the
$T=1, T_{z}= \pm 1$ states. While the former states are shifted due to TO polarization, the latter ones - the $I=0$ ground states of e-e $N-Z= \pm 2$ isotopes - are not influenced by TO effects due to time-reversal symmetry conservation. Hence, the TO terms introduce a very specific source of the isospin symmetry violation in the intrinsic system.

Moreover, as shown in the lower part of Fig. 1, the isospin projected DFT approach always yields ground states with $T=0$ in o-o $N=Z$ nuclei. This is at variance with empirical data; indeed, it is well known (see, e.g., Refs. [33, 34, 35]) that, with two exceptions, the isospin of the g.s. changes from $T=0$ in light $(A<40)$ o-o $N=Z$ nuclei to $T=1$ in heavier $(A>40)$ o-o $N=Z$ nuclei. To be able to address this question in the nuclear DFT, one has to consider simultaneous isospin and angular-momentum projections and also consider residual proton-neutron interactions between valence particles (either perturbatively or by breaking the proton-neutron symmetry). This subject will be considered in forthcoming studies. The focus of this paper is on high-spin superdeformed and terminating states, which - due to large spin polarization and high seniority - are expected to be less affected by the effects mentioned above.

\section{THEORY}

\section{A. Isospin projection formalism}

Within the MF approximation, the isospin symmetry breaking has two sources. The spontaneous breaking of isospin associated with the MF approximation itself [26, 29, 36] is, in this theory, intertwined with the explicit symmetry breaking due to the Coulomb interaction. The essence of our method [28, 29] is to retain the explicit isospin mixing. It is achieved by rediagonalizing the total effective nuclear Hamiltonian $\hat{H}_{N N}$ in good-isospin basis. The basis is generated by using the projection-after-variation technique, that is, by acting with the standard one-dimensional isospin-projection operator on the MF product state $|\Phi\rangle$ :

$$
\begin{aligned}
\left|T T_{z}\right\rangle & =\frac{1}{\sqrt{N_{T T_{z}}}} \hat{P}_{T_{z} T_{z}}^{T}|\Phi\rangle \\
& =\frac{2 T+1}{2 \sqrt{N_{T T_{z}}}} \int_{0}^{\pi} d \beta \sin \beta d_{T_{z} T_{z}}^{T}(\beta) \hat{R}(\beta)|\Phi\rangle,(1)
\end{aligned}
$$

where $\beta$ denotes the Euler angle associated with the rotation operator $\hat{R}(\beta)=e^{-i \beta \hat{T}_{y}}$ about the $y$-axis in the isospace, $d_{T_{z} T_{z}}^{T}(\beta)$ is the Wigner function [37], and $T_{z}=(N-Z) / 2$ is the third component of the total isospin $T$. The wave function $|\Phi\rangle$ is obtained self-consistently by solving the Skyrme-Hartree-Fock (SHF) equations. In the HF limit, the $z$-component $T_{z}$ is strictly conserved. The overlap $N_{T T_{z}}$, or interchangeably the normalization 
factor $b_{T, T_{z}}$, are given by

$$
\begin{aligned}
N_{T T_{z}} & \equiv b_{T, T_{z}}^{2}=\left\langle\Phi\left|\hat{P}_{T_{z} T_{z}}^{T}\right| \Phi\right\rangle \\
& =\frac{2 T+1}{2} \int_{0}^{\pi} d \beta \sin \beta d_{T_{z} T_{z}}^{T}(\beta) \mathcal{N}(\beta),
\end{aligned}
$$

where

$$
\mathcal{N}(\beta)=\langle\Phi|\hat{R}(\beta)| \Phi\rangle
$$

stands for the overlap kernel. The good-isospin basis is spanned by the states $\left|T, T_{z}\right\rangle$ that have tangible contributions to the MF state

$$
|\Phi\rangle=\sum_{T \geq\left|T_{z}\right|} b_{T, T_{z}}\left|T, T_{z}\right\rangle, \quad \sum_{T \geq\left|T_{z}\right|}\left|b_{T, T_{z}}\right|^{2}=1 .
$$

In practice, we retain states having $\left|b_{T, T_{z}}\right|^{2}>10^{-10}$, which sets the limit of $T \leq\left|T_{z}\right|+5$.

The total nuclear Hamiltonian consists of the kinetic energy $\hat{T}$, the Skyrme interaction $\hat{V}^{S}$ and the Coulomb interaction $\hat{V}^{C}$ that breaks isospin:

$$
\begin{aligned}
\hat{H}_{N N} & =\hat{T}+\hat{V}^{S}+\hat{V}^{C} \\
& \equiv \hat{T}+\hat{V}^{S}+\hat{V}_{00}^{C}+\hat{V}_{10}^{C}+\hat{V}_{20}^{C},
\end{aligned}
$$

where

$$
\begin{aligned}
& \hat{V}_{00}^{C}\left(r_{i j}\right)=\frac{1}{4} \frac{e^{2}}{r_{i j}}\left(1+\frac{1}{3} \hat{\tau}^{(i)} \circ \hat{\tau}^{(j)}\right) \\
& \hat{V}_{10}^{C}\left(r_{i j}\right)=-\frac{1}{4} \frac{e^{2}}{r_{i j}}\left(\hat{\tau}_{10}^{(i)}+\hat{\tau}_{10}^{(j)}\right) \\
& \hat{V}_{20}^{C}\left(r_{i j}\right)=\frac{1}{4} \frac{e^{2}}{r_{i j}}\left(\hat{\tau}_{10}^{(i)} \hat{\tau}_{10}^{(j)}-\frac{1}{3} \hat{\tau}^{(i)} \circ \hat{\tau}^{(j)}\right)
\end{aligned}
$$

are, respectively, isoscalar, covariant rank-1 (isovector), and covariant rank-2 axial (isotensor) spherical tensor components of the Coulomb interaction. They are constructed by coupling spherical components of the onebody isospin operator:

$$
\hat{\tau}_{10}=\hat{\tau}_{z}, \quad \hat{\tau}_{1 \pm 1}=\mp \frac{1}{\sqrt{2}}\left(\hat{\tau}_{x} \pm i \hat{\tau}_{y}\right)
$$

where $\hat{\tau}_{i}, i=x, y, z$ denote Pauli matrices and symbol $\circ$ stands for the scalar product of isovectors.

The Coulomb interaction is the only source of the isospin symmetry violation in our model. Charge symmetry breaking components of the strong interaction and the isovector kinetic energy (which is quenched as compared to its isoscalar counterpart by a factor $\frac{\Delta M}{M} \sim 0.001$ due to very small mass difference $\Delta M$ between the neutron and the proton relative to mean nucleonic mass $M$ ) are not taken into account. Hence, the isoscalar part of the total Hamiltonian reads $\hat{H}_{00}=\hat{T}+\hat{V}^{S}+\hat{V}_{00}^{C}$. Its matrix elements can be cast into one-dimensional integrals:

$$
\begin{aligned}
\left\langle T T_{z}\left|\hat{H}_{00}\right| T^{\prime} T_{z}\right\rangle= & \frac{\delta_{T T^{\prime}}}{N_{T T_{z}}}\left\langle\Phi\left|\hat{H}_{00} \hat{P}_{T_{z} T_{z}}^{T}\right| \Phi\right\rangle \\
= & \frac{\delta_{T T^{\prime}}}{N_{T T_{z}}} \frac{2 T+1}{2} \int_{0}^{\pi} d \beta \sin \beta d_{T_{z} T_{z}}^{T}(\beta) \mathcal{H}_{00}(\beta),
\end{aligned}
$$

involving the Hamiltonian kernel

$$
\mathcal{H}_{00}(\beta)=\left\langle\Phi\left|\hat{H}_{00} \hat{R}(\beta)\right| \Phi\right\rangle \text {. }
$$

Calculation of matrix elements of the isovector (7) and isotensor (8) components of the Coulomb interaction is slightly more complicated. It appears, however, that these matrix elements can also be reduced to onedimensional integrals over the Euler angle $\beta$ :

$$
\left\langle T^{\prime} T_{z}\left|\hat{V}_{\lambda 0}^{C}\right| T T_{z}\right\rangle=\frac{1}{\sqrt{N_{T^{\prime} T_{z}} N_{T T_{z}}}} \sum_{\mu=-\lambda}^{\mu=\lambda} I_{\lambda \mu}
$$

where

$$
\begin{gathered}
I_{\lambda \mu}=(-1)^{\mu} C_{T T_{z} \lambda 0}^{T^{\prime} T_{z}} C_{T T_{z}-\mu \lambda \mu}^{T^{\prime} T_{z}} \\
\times \frac{2 T+1}{2} \int_{0}^{\pi} d \beta \sin \beta d_{T_{z}-\mu T_{z}}^{T}(\beta)\left\langle\Phi\left|\hat{V}_{\lambda \mu}^{C} \hat{R}(\beta)\right| \Phi\right\rangle,
\end{gathered}
$$

and symbols $C_{T M \lambda \mu}^{T K}$ stand for the Clebsch-Gordan coefficients [37]. The non-axial $(\mu \neq 0)$ isovector and isotensor components of the Coulomb interaction appearing in Eq. (13) are:

$$
\begin{aligned}
& \hat{V}_{1 \pm 1}^{C}\left(r_{i j}\right)=-\frac{1}{4} \frac{e^{2}}{r_{i j}}\left(\hat{\tau}_{1 \pm 1}^{(i)}+\hat{\tau}_{1 \pm 1}^{(j)}\right) \\
& \hat{V}_{2 \pm 1}^{C}\left(r_{i j}\right)=\frac{1}{4} \frac{e^{2}}{r_{i j}} \frac{1}{\sqrt{3}}\left(\hat{\tau}_{1 \pm 1}^{(i)} \hat{\tau}_{10}^{(j)}+\hat{\tau}_{10}^{(i)} \hat{\tau}_{1 \pm 1}^{(j)}\right) \\
& \hat{V}_{2 \pm 2}^{C}\left(r_{i j}\right)=\frac{1}{4} \frac{e^{2}}{r_{i j}} \sqrt{\frac{2}{3}} \hat{\tau}_{1 \pm 1}^{(i)} \hat{\tau}_{1 \pm 1}^{(j)} .
\end{aligned}
$$

Equations (12) - 13) can be derived by using the standard transformation rules for a covariant spherical-tensor operator $\hat{T}_{\lambda \mu}$ of rank- $\lambda$ under the three-dimensional rotation by the Euler angles $\Omega=(\alpha, \beta, \gamma)$, that is,

$$
\hat{R}(\Omega) \hat{T}_{\lambda \mu} \hat{R}(\Omega)^{\dagger}=\sum_{\mu^{\prime}} D_{\mu^{\prime} \mu}^{\lambda}(\Omega) \hat{T}_{\lambda \mu^{\prime}}
$$

where the $\mathrm{SO}(3)$ projection operator $\hat{P}_{K M}^{T}(\Omega)$ commutes with spherical tensors as [38]

$$
\begin{aligned}
& \hat{P}_{K_{f} M_{f}}^{T_{f}} \hat{T}_{\lambda \mu} \hat{P}_{M_{i} K_{i}}^{T_{i}}= \\
= & C_{T_{i} M_{i} \lambda \mu}^{T_{f} M_{f}} \sum_{M \mu^{\prime}}(-1)^{\left(\mu^{\prime}-\mu\right)} C_{T_{i} M \lambda \mu^{\prime}}^{T_{f} K_{f}} \hat{T}_{\lambda \mu^{\prime}} \hat{P}_{M K_{i}}^{T_{i}}
\end{aligned}
$$

The cornerstone of the isospin projection scheme described above is a calculation of the Hamiltonian and norm kernels and subsequent one-dimensional integration over the Euler angle $\beta$. The integrals are calculated numerically using the Gauss-Legandre quadrature, which is very well suited for this problem provided that the calculated kernels are non-singular. 


\section{B. Inverse of the overlap matrix}

A prerequisite for the calculation of the integrals in Eqs. (2), (10), and (13) is the isospin rotation of the Slater determinant. To this end, one has to perform an independent rotation of s.p. neutron,

$$
\varphi_{i}=\left(\begin{array}{c}
\varphi_{i}^{(n)} \\
0
\end{array}\right), \quad i=1,2, \ldots, N
$$

and proton,

$$
\varphi_{i}=\left(\begin{array}{c}
0 \\
\varphi_{i}^{(p)}
\end{array}\right), \quad i=N+1, \ldots, A,
$$

HF wave functions. The use of the two-dimensional (spinor) representation of isospin constitutes a natural formalism for the isospin projection technique. In this representation, the upper (neutron, $q=1$ ) and lower (proton, $q=-1$ ) components of the isospin-rotated states are given by:

$$
\hat{R}(\beta) \varphi_{i} \equiv \tilde{\varphi}_{i}(\beta)
$$

where

$$
\begin{aligned}
& \tilde{\varphi}_{i}(\beta)=\left(\begin{array}{ll}
\varphi_{i}^{(n)} & \cos \frac{\beta}{2} \\
\varphi_{i}^{(n)} & \sin \frac{\beta}{2}
\end{array}\right), \quad i=1,2, \ldots, N, \\
& \tilde{\varphi}_{i}(\beta)=\left(\begin{array}{cc}
-\varphi_{i}^{(p)} & \sin \frac{\beta}{2} \\
\varphi_{i}^{(p)} & \cos \frac{\beta}{2}
\end{array}\right), \quad i=N+1, \ldots, A
\end{aligned}
$$

To calculate the kernel $\langle\Phi|\hat{\mathcal{Q}}| \tilde{\Phi}\rangle$ of an arbitrary operator $\hat{\mathcal{Q}}$ between two non-orthogonal Slater determinants, we apply the generalized Wick's theorem, see, e.g., Ref. [39]. In particular, the norm kernel can be written in a compact form:

$$
\langle\Phi \mid \tilde{\Phi}\rangle=\operatorname{Det} \tilde{O}
$$

where the overlap matrix is:

$$
\tilde{O}(\beta)_{i j}=\int d \boldsymbol{r} \sum_{\sigma, q} \varphi_{i}^{*}(\boldsymbol{r}, \sigma, q) \tilde{\varphi}_{j}(\boldsymbol{r}, \sigma, q, \beta) .
$$

To calculate kernels appearing in the projection formalism, one needs to invert the overlap matrix $\tilde{O}^{-1}$. This can cause serious problems due to the presence of singularities [22, 40]. The regularization of kernel singularities is a difficult problem [23]. Thus far, a regularization scheme has been worked out only for a very specific class of functionals (or effective density-dependent interactions) solely involving integer powers of local densities 23]. Unfortunately, almost all commonly used Skyrme and Gogny parameterizations, except for SIII [41], involve fractional powers of the density. The appearance of singularities prevents us from using the local Slater approximation for the Coulomb exchange. In the present work we treat it exactly using the method of the Gaussian decomposition of the Coulomb interaction, as described in Refs. [42, 43].

Compared to the particle number or the angular momentum projection schemes, isospin projection is a relatively simple procedure. In particular, the dependence of the inverse matrix $\tilde{O}^{-1}(\beta)$ on the isorotation angle $\beta$ can be determined analytically and this enables us to demonstrate that the isospin projection is free from kernel singularities. To this end, we write the overlap matrix (25) in the form:

$$
\tilde{O}(\beta)=\left(\begin{array}{cc}
\cos \frac{\beta}{2} I_{N} & -\sin \frac{\beta}{2} O \\
\sin \frac{\beta}{2} O^{\dagger} & \cos \frac{\beta}{2} I_{Z}
\end{array}\right)
$$

where $I_{N}\left(I_{Z}\right)$ stands for the $N \times N(Z \times Z)$ unit matrix, while $O=\left\langle\varphi^{(n)} \mid \varphi^{(p)}\right\rangle$ is the rectangular $N \times Z$ overlap matrix of the neutron and proton s.p. wave functions. Using the singular value decomposition (SVD) technique, the matrix $O$ can be written as:

$$
O=W D V^{\dagger}
$$

where $W$ is an $N \times Z$ rectangular matrix having orthogonal columns $\left(W^{\dagger} W=I_{Z}\right), D$ is a diagonal $Z \times Z$ real and non-negative matrix $\left(D^{*}=D\right)$, and $V$ is a $Z \times Z$ quadratic unitary matrix $\left(V^{\dagger} V=V V^{\dagger}=I_{Z}\right)$. The SVD decomposition (27) further implies that:

$$
\begin{aligned}
& O O^{\dagger}=W D^{2} W^{\dagger} \\
& O^{\dagger} O=V D^{2} V^{\dagger}
\end{aligned}
$$

where $O O^{\dagger}$ and $O^{\dagger} O$ are matrices of dimension $N \times N$ and $Z \times Z$, respectively.

The SVD decomposition allows us to analytically diagonalize the overlap matrix $\tilde{O}(\beta)$. Without loss of generality we assume that $N \geq Z$. Hence, the product $\tilde{O}(-\beta) \tilde{O}(\beta)$ can be written as:

$$
\tilde{O}(-\beta) \tilde{O}(\beta)=\left(\begin{array}{cc}
\tilde{W} & 0 \\
0 & V
\end{array}\right)\left(\begin{array}{cc}
\cos ^{2} \frac{\beta}{2} I_{N}+\sin ^{2} \frac{\beta}{2} \tilde{D}^{2} & 0 \\
0 & \cos ^{2} \frac{\beta}{2} I_{Z}+\sin ^{2} \frac{\beta}{2} D^{2}
\end{array}\right)\left(\begin{array}{cc}
\tilde{W} & 0 \\
0 & V
\end{array}\right)^{\dagger}
$$


where $\tilde{W}$ of dimension $N \times N$ is a unitary matrix composed of columns $W$ :

$$
\tilde{W}=(W, \bar{W}) \quad \text { where } \quad \tilde{W} \tilde{W}^{\dagger}=\tilde{W}^{\dagger} \tilde{W}=I_{N},
$$

and $\bar{W}$ is the unitary complement.

The $\tilde{D}$ matrix, on the other hand, is the $Z \times Z$ matrix $D$ completed to the dimension $N \times N$ by zeros:

$$
\tilde{D}=\left(\begin{array}{cc}
D & 0 \\
0 & 0
\end{array}\right)
$$

$$
\tilde{O}(\beta)^{-1}=\left(\begin{array}{cc}
\tilde{W} & 0 \\
0 & V
\end{array}\right)\left(\begin{array}{c}
\frac{1}{\cos ^{2} \frac{\beta}{2} I_{N}+\sin ^{2} \frac{\beta}{2} \tilde{D}^{2}} \\
0
\end{array}\right.
$$

This expression shows explicitly that the inverse of the overlap matrix $\tilde{O}(\beta)_{i j}^{-1}$ can be singular only if $\beta=\pi$. Indeed, in the case of $N=Z$, the $i$ th denominator becomes zero,

$$
\left(\cos ^{2} \frac{\beta}{2} I_{N}+\sin ^{2} \frac{\beta}{2} \tilde{D}^{2}\right)_{i}=0,
$$

only when $\beta=\pi$ and the corresponding singular value vanishes: $D_{i}=0$. In the case of $N \neq Z$, the only difference is that the matrix $\tilde{D}$ contains zeros by construction, see Eq. (31). Fortunately, for the isospin projection, the singularity at $\beta=\pi$ is compensated by the Jacobian $\sin \beta d \beta$.

\section{Isospin structure of the density matrix}

To calculate the transition density matrix and determine its isotopic structure it is convenient to introduce auxiliary ket-states defined as:

$$
\tilde{\phi}_{i}(\boldsymbol{x}, q, \beta) \equiv \sum_{j=1}^{A} \tilde{\varphi}_{j}(\boldsymbol{x}, q, \beta) \tilde{O}(\beta)_{j i}^{-1} .
$$

where the space-spin coordinates are abbreviated as $\boldsymbol{x}=$ $(\boldsymbol{r}, \sigma)$. This allows for rewriting the transition density matrix into a diagonal form:

$$
\tilde{\rho}\left(\boldsymbol{x}, q ; \boldsymbol{x}^{\prime}, q^{\prime}\right) \equiv \sum_{i=1}^{A} \varphi_{i}^{*}\left(\boldsymbol{x}^{\prime}, q^{\prime}\right) \tilde{\phi}_{i}(\boldsymbol{x}, q, \beta),
$$

where the summation runs over a set of s.p. states, in full analogy with the HF particle density matrix. To alleviate the notation, in what follows we do not indicate the dependence of the transition density matrices on the isorotation angle $\beta$.
Since the first and third matrices on the right hand side of Eq. (29) are unitary and the second matrix is diagonal, the inverse of the overlap matrix reads:

An arbitrary matrix in the isospace can be decomposed in terms of the isospin Pauli matrices. In particular, the transition density matrix $\tilde{\rho}\left(\boldsymbol{x}, q ; \boldsymbol{x}^{\prime}, q^{\prime}\right)$ can be expressed as:

$$
\begin{aligned}
\tilde{\rho}\left(\boldsymbol{x}, q ; \boldsymbol{x}^{\prime}, q^{\prime}\right) & =\frac{1}{2} \tilde{\rho}_{00}\left(\boldsymbol{x}, \boldsymbol{x}^{\prime}\right) \delta_{q q^{\prime}} \\
& +\frac{1}{2} \sum_{\alpha=0, \pm 1} \tau_{1 \alpha}\left(q, q^{\prime}\right) \tilde{\rho}_{1 \alpha}\left(\boldsymbol{x}, \boldsymbol{x}^{\prime}\right),
\end{aligned}
$$

where

$$
\begin{array}{r}
\tau_{10}\left(q, q^{\prime}\right)=\left\langle q\left|\hat{\tau}_{10}\right| q^{\prime}\right\rangle=q \delta_{q, q^{\prime}}, \\
\tau_{1 \pm 1}\left(q, q^{\prime}\right)=\left\langle q\left|\hat{\tau}_{1 \pm 1}\right| q^{\prime}\right\rangle=\mp \sqrt{2} \delta_{q, \pm 1} \delta_{q^{\prime}, \mp 1} .
\end{array}
$$

The isoscalar $\tilde{\rho}_{00}$ and isovector $\tilde{\rho}_{1 \alpha}$ transition density matrices are calculated by contracting $\tilde{\rho}$ :

$$
\begin{aligned}
\tilde{\rho}_{00} & =\operatorname{Tr} \tilde{\rho}, \\
\tilde{\rho}_{10} & =\operatorname{Tr}\left(\tau_{10} \tilde{\rho}\right), \\
\tilde{\rho}_{1 \pm 1} & =-\operatorname{Tr}\left(\tau_{1 \mp 1} \tilde{\rho}\right) .
\end{aligned}
$$

The use of the two-dimensional spinor notation is convenient for analytical derivations. However, from the numerical-programming perspective it is better to present expressions in a one-dimensional form, because it allows us to exploit the separability of proton and neutron wave functions and densities - a common feature of essentially all the existing HF codes including the HFODD solver used in this work.

To write the isoscalar and isovector densities in the one-dimensional form we introduce two sets of neutronlike $(i=1,2, \ldots, N)$ wave functions:

$$
\begin{aligned}
& \tilde{\phi}_{i}^{(n)}(\boldsymbol{x}) \equiv \sum_{j=1}^{N} \varphi_{j}^{(n)}(\boldsymbol{x}) \tilde{O}(\beta)_{j i}^{-1}, \\
& \tilde{\phi}_{i}^{(p)}(\boldsymbol{x}) \equiv \sum_{j=N+1}^{A} \varphi_{j}^{(p)}(\boldsymbol{x}) \tilde{O}(\beta)_{j i}^{-1},
\end{aligned}
$$


and two sets of proton-like $(i=N+1, N+2, \ldots, A)$ wave functions:

$$
\begin{aligned}
\tilde{\phi}_{i}^{(n)}(\boldsymbol{x}) & \equiv \sum_{j=1}^{N} \varphi_{j}^{(n)}(\boldsymbol{x}) \tilde{O}(\beta)_{j i}^{-1}, \\
\tilde{\phi}_{i}^{(p)}(\boldsymbol{x}) & \equiv \sum_{j=N+1}^{A} \varphi_{j}^{(p)}(\boldsymbol{x}) \tilde{O}(\beta)_{j i}^{-1} .
\end{aligned}
$$

Again, the dependence of these states on the isorotation angle $\beta$ is not explicitly indicated. Using these wave functions, we define two neutron-like densities:

$$
\begin{aligned}
& \tilde{\rho}_{n}^{(n)}\left(\boldsymbol{x}, \boldsymbol{x}^{\prime}\right)=\sum_{i=1}^{N} \varphi_{i}^{(n) *}\left(\boldsymbol{x}^{\prime}\right) \tilde{\phi}_{i}^{(n)}(\boldsymbol{x}) \\
& \tilde{\rho}_{n}^{(p)}\left(\boldsymbol{x}, \boldsymbol{x}^{\prime}\right)=\sum_{i=1}^{N} \varphi_{i}^{(n) *}\left(\boldsymbol{x}^{\prime}\right) \tilde{\phi}_{i}^{(p)}(\boldsymbol{x}),
\end{aligned}
$$

and two proton-like densities:

$$
\begin{aligned}
& \tilde{\rho}_{p}^{(n)}\left(\boldsymbol{x}, \boldsymbol{x}^{\prime}\right)=\sum_{i=N+1}^{A} \varphi_{i}^{(p) *}\left(\boldsymbol{x}^{\prime}\right) \tilde{\phi}_{i}^{(n)}(\boldsymbol{x}) \\
& \tilde{\rho}_{p}^{(p)}\left(\boldsymbol{x}, \boldsymbol{x}^{\prime}\right)=\sum_{i=N+1}^{A} \varphi_{i}^{(p) *}\left(\boldsymbol{x}^{\prime}\right) \tilde{\phi}_{i}^{(p)}(\boldsymbol{x}) .
\end{aligned}
$$

In these definitions, subscripts indicate whether the density is neutron- or proton-like and superscripts indicate whether the summation indices in Eqs. (40)-(43) run over neutron or proton states. The densities $\tilde{\rho}_{n}^{(p)}\left(\boldsymbol{x}, \boldsymbol{x}^{\prime}\right)$ and $\tilde{\rho}_{p}^{(n)}\left(\boldsymbol{x}, \boldsymbol{x}^{\prime}\right)$ can be associated, respectively, with the raising and lowering components of the analog spin [44, 45].

The isoscalar and isovector densities (39) can now be expressed by using the auxiliary densities (44)-(47), and they read

$$
\begin{aligned}
\tilde{\rho}_{00}\left(\boldsymbol{x}, \boldsymbol{x}^{\prime}\right) & =\cos \frac{\beta}{2}\left(\tilde{\rho}_{n}^{(n)}\left(\boldsymbol{x}, \boldsymbol{x}^{\prime}\right)+\tilde{\rho}_{p}^{(p)}\left(\boldsymbol{x}, \boldsymbol{x}^{\prime}\right)\right) \\
& -\sin \frac{\beta}{2}\left(\tilde{\rho}_{n}^{(p)}\left(\boldsymbol{x}, \boldsymbol{x}^{\prime}\right)-\tilde{\rho}_{p}^{(n)}\left(\boldsymbol{x}, \boldsymbol{x}^{\prime}\right)\right) \\
\tilde{\rho}_{10}\left(\boldsymbol{x}, \boldsymbol{x}^{\prime}\right)= & \cos \frac{\beta}{2}\left(\tilde{\rho}_{n}^{(n)}\left(\boldsymbol{x}, \boldsymbol{x}^{\prime}\right)-\tilde{\rho}_{p}^{(p)}\left(\boldsymbol{x}, \boldsymbol{x}^{\prime}\right)\right) \\
- & \sin \frac{\beta}{2}\left(\tilde{\rho}_{n}^{(p)}\left(\boldsymbol{x}, \boldsymbol{x}^{\prime}\right)+\tilde{\rho}_{p}^{(n)}\left(\boldsymbol{x}, \boldsymbol{x}^{\prime}\right)\right) \\
\tilde{\rho}_{11}\left(\boldsymbol{x}, \boldsymbol{x}^{\prime}\right)= & -\sqrt{2} \cos \frac{\beta}{2} \tilde{\rho}_{p}^{(n)}\left(\boldsymbol{x}, \boldsymbol{x}^{\prime}\right) \\
& +\sqrt{2} \sin \frac{\beta}{2} \tilde{\rho}_{p}^{(p)}\left(\boldsymbol{x}, \boldsymbol{x}^{\prime}\right) \\
\tilde{\rho}_{1-1}\left(\boldsymbol{x}, \boldsymbol{x}^{\prime}\right) & =\sqrt{2} \cos \frac{\beta}{2} \tilde{\rho}_{n}^{(p)}\left(\boldsymbol{x}, \boldsymbol{x}^{\prime}\right) \\
& +\sqrt{2} \sin \frac{\beta}{2} \tilde{\rho}_{n}^{(n)}\left(\boldsymbol{x}, \boldsymbol{x}^{\prime}\right) .
\end{aligned}
$$

\section{Hamiltonian kernel}

Conventional Skyrme EDF can be expressed by bilinear forms of six isoscalar $(t=0)$ and six isovector $(t=1)$ local densities, including the particle $\rho_{t}$, kinetic $\tau_{\tau}$, spin $\boldsymbol{s}_{t}$, spin-kinetic $\boldsymbol{T}_{t}$, current $\boldsymbol{j}_{t}$, and spin-current $\stackrel{\leftrightarrow}{J}_{t}$ densities and their derivatives. Standard definitions of these densities can be found in numerous references, see, e.g., Refs. 9, 46] and references quoted therein. It is to be noted that in the standard MF theory the proton and neutron s.p. wave functions are not mixed, i.e., the proton-neutron symmetry is strictly conserved [46, 47]. Therefore, the MF isoscalar (isovector) densities are simply sums (differences) of neutron and proton densities since only the third component of the isovector density is nonzero.

The isospin projection essentially preserves the functional form of the Skyrme EDF derived by averaging the isospin-invariant Skyrme interaction over the Slater determinant. All what needs to be done is a replacement of the local density matrix by the corresponding transition density matrix. Moreover, the bilinear terms that depend of the isovector densities must be replaced by the full isoscalar products of the corresponding isovector transition densities. Special care should be taken of the density-dependent term of the Skyrme interaction as the extension of this term to the transition case is undefined in the process of averaging the Skyrme interaction over the Slater determinant. Following the argumentation of Refs. [48, 49], we replace the isoscalar density by the transition isoscalar density matrix, that is, $\rho_{n}+\rho_{p} \longrightarrow \tilde{\rho}_{00}$.

As for the Coulomb-interaction kernel, it depends on the isoscalar and isovector transition densities in the following way:

$$
\begin{gathered}
\left\langle\Phi\left|\hat{V}^{C} \hat{R}(\beta)\right| \Phi\right\rangle= \\
=\frac{e^{2}}{4} \int d \boldsymbol{r}_{1} \int d \boldsymbol{r}_{2} \frac{1}{r_{12}} \sum_{\lambda=0}^{2} \sum_{\mu=-\lambda}^{\lambda} \mathcal{V}_{\lambda \mu}^{C}\left(\boldsymbol{r}_{1}, \boldsymbol{r}_{2}\right),
\end{gathered}
$$

where 


$$
\begin{aligned}
\mathcal{V}_{00}^{C}= & \frac{1}{2}\left\{\tilde{\rho}_{00}\left(\boldsymbol{r}_{1}\right) \tilde{\rho}_{00}\left(\boldsymbol{r}_{2}\right)+\frac{1}{3} \tilde{\rho}_{1}\left(\boldsymbol{r}_{1}\right) \circ \tilde{\rho}_{1}\left(\boldsymbol{r}_{2}\right)\right\} \\
- & \frac{1}{4}\left\{\tilde{\rho}_{00}\left(\boldsymbol{r}_{2}, \boldsymbol{r}_{1}\right) \tilde{\rho}_{00}\left(\boldsymbol{r}_{1}, \boldsymbol{r}_{2}\right)+\frac{1}{3} \tilde{\rho}_{1}\left(\boldsymbol{r}_{2}, \boldsymbol{r}_{1}\right) \circ \tilde{\rho}_{1}\left(\boldsymbol{r}_{1}, \boldsymbol{r}_{2}\right)\right. \\
& \left.+\tilde{\boldsymbol{s}}_{00}\left(\boldsymbol{r}_{2}, \boldsymbol{r}_{1}\right) \cdot \tilde{\boldsymbol{s}}_{00}\left(\boldsymbol{r}_{1}, \boldsymbol{r}_{2}\right)+\frac{1}{3} \tilde{\boldsymbol{s}}_{1}\left(\boldsymbol{r}_{2}, \boldsymbol{r}_{1}\right) \circ \tilde{\boldsymbol{s}}_{1}\left(\boldsymbol{r}_{1}, \boldsymbol{r}_{2}\right)\right\}, \\
\mathcal{V}_{10}^{C}= & -\tilde{\rho}_{00}\left(\boldsymbol{r}_{1}\right) \tilde{\rho}_{10}\left(\boldsymbol{r}_{2}\right)+\frac{1}{2}\left\{\tilde{\rho}_{00}\left(\boldsymbol{r}_{2}, \boldsymbol{r}_{1}\right) \tilde{\rho}_{10}\left(\boldsymbol{r}_{1}, \boldsymbol{r}_{2}\right)+\tilde{\boldsymbol{s}}_{00}\left(\boldsymbol{r}_{2}, \boldsymbol{r}_{1}\right) \cdot \tilde{\boldsymbol{s}}_{10}\left(\boldsymbol{r}_{1}, \boldsymbol{r}_{2}\right)\right\}, \\
\mathcal{V}_{1 \pm 1}^{C}= & \tilde{\rho}_{00}\left(\boldsymbol{r}_{1}\right) \tilde{\rho}_{1 \mp 1}\left(\boldsymbol{r}_{2}\right)-\frac{1}{2}\left\{\tilde{\rho}_{00}\left(\boldsymbol{r}_{2}, \boldsymbol{r}_{1}\right) \tilde{\rho}_{1 \mp 1}\left(\boldsymbol{r}_{1}, \boldsymbol{r}_{2}\right)+\tilde{\boldsymbol{s}}_{00}\left(\boldsymbol{r}_{2}, \boldsymbol{r}_{1}\right) \cdot \tilde{\boldsymbol{s}}_{1 \mp 1}\left(\boldsymbol{r}_{1}, \boldsymbol{r}_{2}\right)\right\}, \\
\mathcal{V}_{20}^{C}= & \frac{1}{3}\left\{\tilde{\rho}_{10}\left(\boldsymbol{r}_{1}\right) \tilde{\rho}_{10}\left(\boldsymbol{r}_{2}\right)+\tilde{\rho}_{11}\left(\boldsymbol{r}_{1}\right) \tilde{\rho}_{1-1}\left(\boldsymbol{r}_{2}\right)\right\} \\
- & \frac{1}{6}\left\{\tilde{\rho}_{10}\left(\boldsymbol{r}_{2}, \boldsymbol{r}_{1}\right) \tilde{\rho}_{10}\left(\boldsymbol{r}_{1}, \boldsymbol{r}_{2}\right)+\tilde{\rho}_{11}\left(\boldsymbol{r}_{2}, \boldsymbol{r}_{1}\right) \tilde{\rho}_{1-1}\left(\boldsymbol{r}_{1}, \boldsymbol{r}_{2}\right)\right. \\
& \left.+\tilde{\boldsymbol{s}}_{10}\left(\boldsymbol{r}_{2}, \boldsymbol{r}_{1}\right) \cdot \tilde{\boldsymbol{s}}_{10}\left(\boldsymbol{r}_{1}, \boldsymbol{r}_{2}\right)+\tilde{\boldsymbol{s}}_{11}\left(\boldsymbol{r}_{2}, \boldsymbol{r}_{1}\right) \cdot \tilde{\boldsymbol{s}}_{1-1}\left(\boldsymbol{r}_{1}, \boldsymbol{r}_{2}\right)\right\}, \\
\mathcal{V}_{2 \pm 1}^{C}= & -\frac{1}{\sqrt{3}} \tilde{\rho}_{10}\left(\boldsymbol{r}_{1}\right) \tilde{\rho}_{1 \mp 1}\left(\boldsymbol{r}_{2}\right)+\frac{1}{2 \sqrt{3}}\left\{\tilde{\rho}_{10}\left(\boldsymbol{r}_{2}, \boldsymbol{r}_{1}\right) \tilde{\rho}_{1 \mp 1}\left(\boldsymbol{r}_{1}, \boldsymbol{r}_{2}\right)+\tilde{\boldsymbol{s}}_{10}\left(\boldsymbol{r}_{2}, \boldsymbol{r}_{1}\right) \cdot \tilde{\boldsymbol{s}}_{1 \mp 1}\left(\boldsymbol{r}_{1}, \boldsymbol{r}_{2}\right)\right\}, \\
\mathcal{V}_{2 \pm 2}^{C}= & \frac{1}{\sqrt{6}} \tilde{\rho}_{1 \mp 1}\left(\boldsymbol{r}_{1}\right) \tilde{\rho}_{1 \mp 1}\left(\boldsymbol{r}_{2}\right)-\frac{1}{2 \sqrt{6}}\left\{\tilde{\rho}_{1 \mp 1}\left(\boldsymbol{r}_{2}, \boldsymbol{r}_{1}\right) \tilde{\rho}_{1 \mp 1}\left(\boldsymbol{r}_{1}, \boldsymbol{r}_{2}\right)+\tilde{\boldsymbol{s}}_{1 \mp 1}\left(\boldsymbol{r}_{2}, \boldsymbol{r}_{1}\right) \cdot \tilde{\boldsymbol{s}}_{1 \mp 1}\left(\boldsymbol{r}_{1}, \boldsymbol{r}_{2}\right)\right\} .
\end{aligned}
$$

Here, the symbols $\cdot$ and $\circ$ stand for the scalar products of vectors and isovectors, respectively.

\section{E. Coulomb rediagonalization and the isospin mixing}

To properly account for the isospin mixing effects, following Refs. 28, 29], the total Hamiltonian $\hat{H}_{N N}$ (5) (strong interaction plus the Coulomb interaction) is rediagonalized in the space spanned by the good-isospin wave functions (10), and the resulting eigenstates are denoted by

$$
\left|n, T_{z}\right\rangle=\sum_{T \geq\left|T_{z}\right|} a_{T, T_{z}}^{n}\left|T, T_{z}\right\rangle
$$

and numbered by index $n$. The amplitudes $a_{T, T_{z}}^{n}$ define the degree of isospin mixing. In particular, the isospinmixing parameter for the lowest energy solution $E_{n=1, T_{z}}$ is defined as $\alpha_{C}=1-\left|a_{\left|T_{z}\right|, T_{z}}^{n=1}\right|^{2}$.

Precise determination of the Coulomb mixing constitutes a notoriously difficult problem, but is strongly motivated by its impact on fundamental physics tested through the super-allowed $\beta$ decays [50, 51, 52]. Of importance here is to capture the proper balance between the short-range strong interaction and the long-range Coulomb polarization. This balance is naturally taken into account by the DFT approach, but is not accessible within a perturbative-analysis theory [53] or hydrodynamical model [54]. This is illustrated in Fig. 3 that shows the excitation energies of the doorway $T=1$ states in e-e $N=Z$ nuclei, i.e., the energies of the $n=2$ states

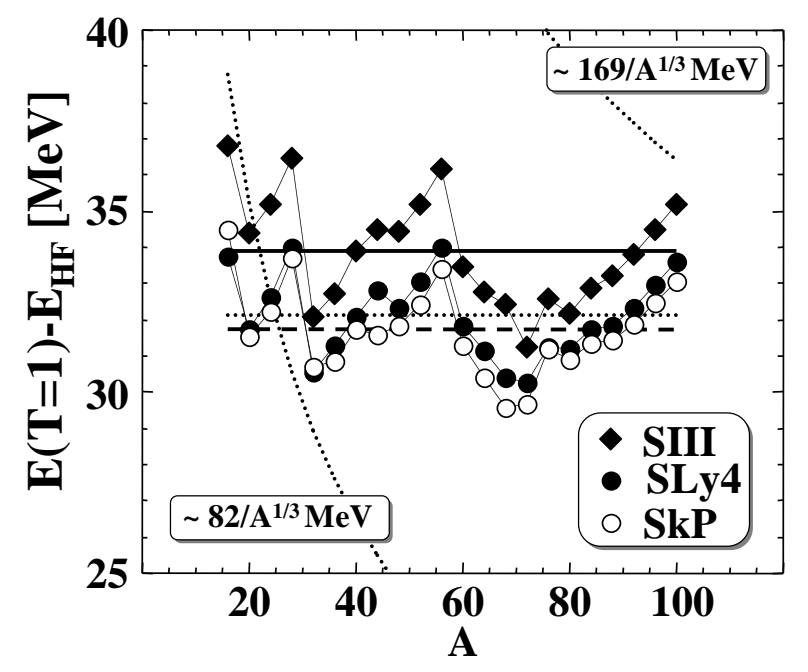

FIG. 3: Excitation energies of the doorway $T=1$ states in the e-e $N=Z$ nuclei relative to the SHF g.s. energies $E_{H F}$. Diamonds, dots, and circles show the self-consistent results obtained in the isospin-projected DFT with SIII [41], SLy4 [55], and $\mathrm{SkP}$ [56] Skyrme functionals, respectively. Horizontal lines mark the mean DFT values. Thick dotted lines show estimates based on the perturbation theory, $E_{T=1} \approx$ $82 / A^{1 / 3} \mathrm{MeV}$ [53, 54], and on the hydrodynamical model, $E_{T=1} \approx 169 / A^{1 / 3} \mathrm{MeV}$ [54].

in Eq. (59) relative to the HF g.s. energies $E_{H F}$. The estimates given by the perturbative/hydrodynamical approaches are clearly at variance with the self-consistent results.

Figure 3 shows that not only the values but also the 


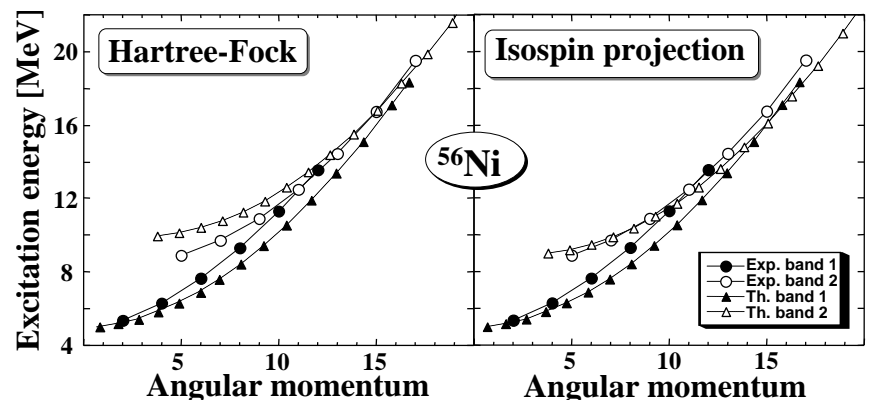

FIG. 4: Superdeformed bands in ${ }^{56} \mathrm{Ni}$. Experimental data for Band 1 (dots) and Band 2 (circles) of Ref. [20] are compared with $\mathrm{HF}+\mathrm{SLy} 4_{L}$ predictions marked by full and open triangles, respectively. The left panel shows the standard HF results while the predicted $T=0 \mathrm{SD}$ bands obtained in the isospin-projected approach are displayed in the right panel.

$A$-dependence of the doorway excitation energies differ substantially from the self-consistent results, pointing very clearly to a non-perturbative origin of the Coulomb mixing. One can also notice that the mean excitation energies of the doorway states (directly impacting the Coulomb mixing) strongly depend on the EDF parameterization. The currently used Skyrme functionals are not sufficiently constrained in the isospin sector to provide reliable estimates of $\alpha_{C}$. At this point is also not at all obvious what parts of EDF have to be refined in order to improve on this situation, see Ref. [29] for further details.

\section{ILLUSTRATIVE EXAMPLES}

All calculations presented in this work were obtained by using isospin projection implemented in the DFT solver HFODD [57, [58]. We used the harmonic-oscillator basis of $N_{0}=12$ shells. We considered SIII [41], SLy4 [55], and SkP [56] Skyrme functionals, as well as for their Landau and tensor versions denoted by subscripts $L$ and $T$, respectively, see Ref. [59] for details. Pairing was neglected.

\section{A. Superdeformed bands in ${ }^{56} \mathrm{Ni}$}

We begin discussion from the case of SD bands in ${ }^{56} \mathrm{Ni}$. As discussed in Sec. III the standard cranked HF theory gives a reasonable reproduction of Band 1 while there are problems with theoretical interpretation of Band 2 built on p-h excitations to the $[440] 1 / 2$ proton and neutron levels. The results are summarized in Fig. 4. The HF (left panel) and isospin-projected (right panel) calculations were performed with SLy $4_{L}$ functional. Since the $\mathrm{HF}$ configuration corresponding to Band 1 is predominantly isospin symmetric, it is only weakly affected by the isospin projection. The calculated isospin impurity within this band is small $(\sim 2 \%)$. This value is similar to that obtained for the spherical g.s. configuration of ${ }^{56} \mathrm{Ni}$. On the other hand, the Band 2 based on the $\pi[440] 1 / 2$ orbital represents, before rediagonalization, almost equal mixture of the $T=0$ and $T=1$ components. Therefore, its isospin impurity assumes an unrealistic value of about $50 \%$.

Following the isospin projection, the low-spin part of the $T=0 \mathrm{SD}$ band originating from Band 2 is shifted down by about $0.95 \mathrm{MeV}$ with respect to the unprojected $\mathrm{HF}$ result. The isospin impurity in this band is in the range of $6 \%$ to $8 \%$, and it slowly increases as a function of the angular momentum. In fact, these values may indicate a presence of an uncontrolled isospin mixing related, most likely, to the angular-momentum non-conservation. As shown schematically in Fig. 2 a $T=1$ SD partner structure is expected to lie higher in energy. We indeed calculate the $T=1$ band (not shown in Fig. 4) to lie about $2.5 \mathrm{MeV}$ above the $T=0$ band. As expected, the $T=0$ and $T=1$ bands projected from the $\nu[440] 1 / 2 \mathrm{MF}$ configuration are almost identical to those projected from the $\pi[440] 1 / 2 \mathrm{MF}$ configuration.

By comparing the results of isospin-projected calculations with experiment, we see a significant improvement as compared to standard HF. The $T=0$ Band 2 agrees well with experiment, both in terms of excitation energy and MoI. The predicted crossing between $T=0$ Bands 1 and 2 occurs around spin $14 \hbar$, i.e., $\sim 2 \hbar$ too high as compared to the data. This discrepancy, however, rather reflects a deficiency of our model in describing the MoI of Band 1, which is slightly overestimated as our calculations neglect pairing correlations that are expected to be important in this configuration [20]. The large energy splitting between the $T=0$ and $T=1$ Band 2 doublet is consistent with observation of only one SD side band. In short, the isospin-projected MF theory provides a quantitative explanation of experimental data on collective band structures in ${ }^{56} \mathrm{Ni}$.

\section{B. Isospin symmetry breaking at band termination}

Terminating states or seniority isomers are fullystretched p-h configurations with a maximum-spin $I_{\max }$ that can be built within a given SM space of valence particles. Because of their simple SM character, terminating states provide a robust probe of SM and MF theories and corresponding effective interactions. In this context, of particular interest are the terminating states associated with the $\left[f_{7 / 2}^{n}\right]_{I_{\max }}$ and $\left[d_{3 / 2}^{-1} f_{7 / 2}^{n+1}\right]_{I_{\max }}$ configurations (in the following, $n$ denotes a number of valence particles outside the ${ }^{40} \mathrm{Ca}$ core) in $20 \leq Z \leq N \leq 24$ nuclei from the lower-fp shell $(A \sim 44)$, which were systematically measured during the last decade 60, 61, 62, 63, 64, 65].

According to MF calculations, these specific states appear to have almost spherical shapes; hence, the correlations resulting from the angular-momentum restoration are practically negligible there [66]. Hence, they can be regarded as extreme cases of an almost undis- 


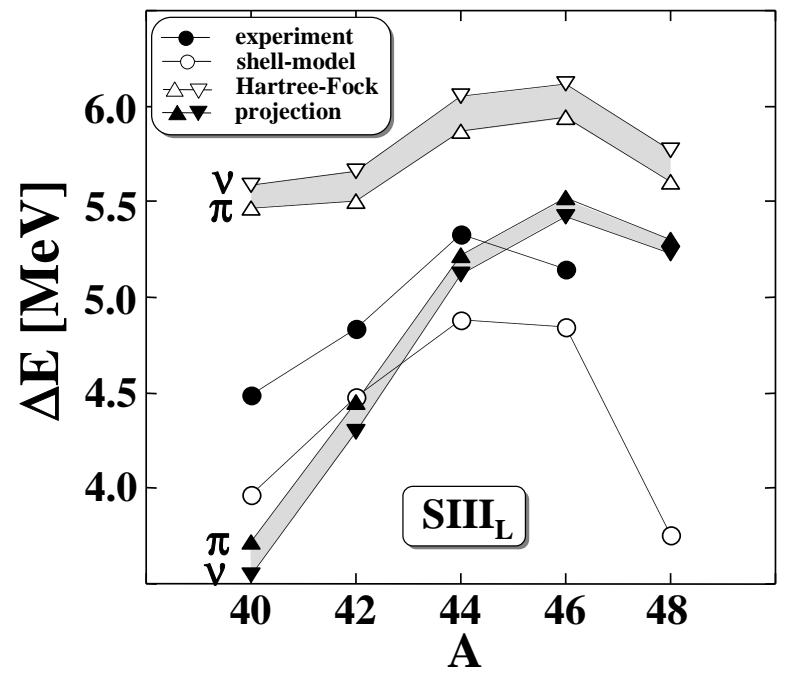

FIG. 5: Energy differences $\Delta E(60)$ between the terminating states in $\mathrm{Ca}, \mathrm{Sc}, \mathrm{Ti}$,and $\mathrm{Cr} N=Z$ nuclei. Open and full triangles mark, respectively, standard and isospin-projected HF results for the $\left[d_{3 / 2}^{-1} f_{7 / 2}^{n+1}\right]_{I_{\max }}^{\pi}$ (up triangles) and $\left[d_{3 / 2}^{-1} f_{7 / 2}^{n+1}\right]_{I_{\max }}^{\nu}$ (down triangles) configurations. Calculations were performed by using the $\mathrm{SIII}_{L}$ functional [59]. Experimental data (dots) and SM results of Ref. 68] (circles) are shown for comparison. Note the limited energy scale.

turbed s.p. motion, thus offering an excellent playground to study, among others, time-odd densities and fields, spin-orbit force [67, 68, 69], tensor interactions [70], and the isospin dependence of cross-shell $(s d-f p)$ p-h matrix elements [68, 71].

While most of the terminating states are uniquely defined, the $\left[d_{3 / 2}^{-1} f_{7 / 2}^{n+1}\right]_{I_{\max }}$ states in the $N=Z$ nuclei provide a notable exception. Indeed, within the MF approximation these particular states can be created by promoting either one proton, $\left[d_{3 / 2}^{-1} f_{7 / 2}^{n+1}\right]_{I_{\text {max }}}^{\pi}$, or one neutron, $\left[d_{3 / 2}^{-1} f_{7 / 2}^{n+1}\right]_{I_{\max }}^{\nu}$, across the magic gap 20, Because the Coulomb energy difference between these configurations is small, the resulting energy levels are almost degenerate: $E\left(\left[d_{3 / 2}^{-1} f_{7 / 2}^{n+1}\right]_{I_{\max }}^{\pi}\right) \approx E\left(\left[d_{3 / 2}^{-1} f_{7 / 2}^{n+1}\right]_{I_{\max }}^{\nu}\right)$. Therefore, we encounter problems related to isospin symmetry similar to those discussed earlier in Sec. [II.

As in the case of ${ }^{56} \mathrm{Ni}$, we encounter the situation where the isospin symmetry is manifestly broken by the MF approximation and the predictions are at variance with empirical data. The difficulty with the isospin content of terminating states was recognized in Ref. [68], where a purely phenomenological method of isospin restoration was proposed. It has resulted in a good MF description of experimental data, at the level of the state-of-the-art SM calculations. Quantitatively, however, the estimated energy correction due to the isospin projection appeared to have surprisingly strong $A$-dependence, changing quite rapidly from $\delta E_{T} \approx 2 \mathrm{MeV}$ in ${ }^{40} \mathrm{Ca}$ down to $\delta E_{T} \approx 1 \mathrm{MeV}$ in ${ }^{46} \mathrm{~V}$, see Fig. 4 of Ref. [68]. This trend has been found to depend weakly on the EDF parameterization.
In the present work, we repeat calculations of Ref. [68], however, by using the mathematically rigorous isospin projection of Sec. [II] The energies of the isospinprojected states $\left[d_{3 / 2}^{-1} f_{7 / 2}^{n+1}\right]_{I_{\max }^{T}=0}^{T}$ are shown in Fig. [5 relative to those of the $\left[f_{7 / 2}^{n}\right]_{I_{\max }}$ configurations:

$$
\Delta E=E\left(\left[d_{3 / 2}^{-1} f_{7 / 2}^{n+1}\right]_{I_{\max }}\right)-E\left(\left[f_{7 / 2}^{n}\right]_{I_{\max }}\right) .
$$

Full up and down triangles show results obtained by projecting the $T=0$ component of the Slater determinant corresponding to either one proton, $\left|\left[d_{3 / 2}^{-1} f_{7 / 2}^{n+1}\right]_{I_{\text {max }}}^{\pi}\right\rangle$, or one neutron, $\left|\left[d_{3 / 2}^{-1} f_{7 / 2}^{n+1}\right]_{I_{\max }}^{\nu}\right\rangle$, p-h excitation through the magic gap 20, respectively. For comparison, the unprojected HF energies for the proton and neutron configurations are shown with the open up and down triangles, respectively. Here, all calculations were performed by using the SIII [41] Skyrme functional.

The isospin-projected results obtained from the $\left|\left[d_{3 / 2}^{-1} f_{7 / 2}^{n+1}\right]_{I_{\max }}^{\pi}\right\rangle$ and $\left|\left[d_{3 / 2}^{-1} f_{7 / 2}^{n+1}\right]_{I_{\max }}^{\nu}\right\rangle$ HF configurations are similar but not identical, reflecting small polarization differences due to proton and neutron p-h excitations. Note, that slightly better results are obtained by projecting from the proton configurations as the resulting levels appear slightly lower in energy. This is probably not surprising, because these states include directly the polarization of the Coulomb field by the proton p-h excitation and thus they have slightly richer isospin structure than their neutron counterparts.

The results of rigorous isospin projection closely follow those obtained in the phenomenological approach of Ref. 68]. As shown in Fig. 6, the energy corrections calculated in the isospin-projected $\mathrm{HF}$,

$$
\delta E_{T}=\frac{1}{2}\left[E\left(\left[d_{3 / 2}^{-1} f_{7 / 2}^{n+1}\right]_{I_{\max }}^{T=1}\right)-E\left(\left[d_{3 / 2}^{-1} f_{7 / 2}^{n+1}\right]_{I_{\max }^{T}}^{T=0}\right)\right],
$$

also exhibit an appreciable $A$-dependence, in a close analogy to the previously obtained phenomenological trend. Consequently, the $A$-dependence of $\Delta E$ (60), calculated using the isospin projected EDF approach also shows a different pattern than experiment and SM. This result is weakly dependent on EDF parameterization, suggesting that there is some generic problem pertaining to the standard form of the Skyrme EDF.

Recently, we have developed a new class of the Skyrme functionals with spin-orbit and tensor terms locally refitted, to reproduce the $f_{7 / 2}-f_{5 / 2}$ spin-orbit splitting in ${ }^{40} \mathrm{Ca},{ }^{48} \mathrm{Ca}$, and ${ }^{56} \mathrm{Ni}[59]$. The spin-orbit and the tensor strengths obtained in this way turned out to be fairly independent of other coupling constants of the Skyrme functional. This result indicates that the strong dependence of the spin-orbit strength on EDF parameterization, in particular on the isoscalar effective mass [72], is likely to be an artifact of fitting protocols based predominantly on data pertaining to bulk nuclear properties. Indeed, as discussed in Ref. [73], the use of inaccurate models in the fitting procedure can lead to results that strongly depend on the fitting protocol itself; hence, can 


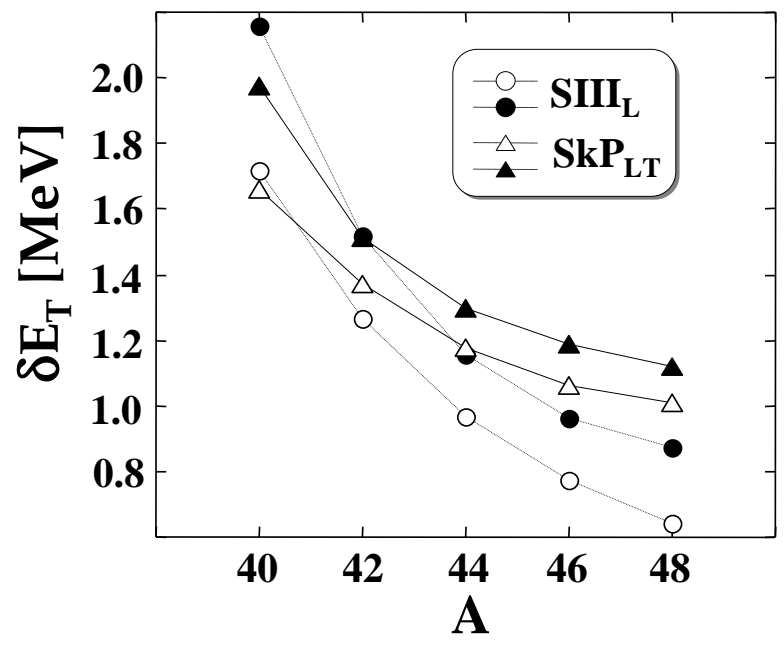

FIG. 6: Full symbols label energy correction $\delta E_{T}$ (61) calculated by projecting good isospin from the HF states corresponding to the $\left[d_{3 / 2}^{-1} f_{7 / 2}^{n+1}\right]_{I_{\max }}^{\pi}$ configurations in $N=Z$ nuclei and subsequent Coulomb rediagonalization. Open symbols represent results of a phenomenological method proposed in Ref. [68]. Calculations were carried out by using the $\mathrm{SIII}_{L}$ (dots) and $\mathrm{SkP}_{L T}$ (triangles) Skyrme functionals [59]. See text for further details.

result in contradictory information on the key model parameters (compare, e.g., results of Refs. [72] and [74]). It seems that this is exactly the case for the current parameterizations of the Skyrme EDF. As shown recently in Ref. 75], parameterizations that correctly describe the spin-orbit properties in light nuclei do not fare well in heavier systems. This again points to limitations of the second-order Skyrme EDF [76] and to a danger of drawing conclusions on tensor interactions from global fits 72.

Applications of new functionals to the terminating states in $N \neq Z, A \sim 44$ nuclei 77 ] have revealed that removal of the artificial isoscalar effective mass scaling from the spin-orbit restores the effective mass scaling in the s.p. level density. As a consequence, only the forces having large isoscalar effective masses $\left(\frac{M^{*}}{M} \geq 0.9\right)$ such as $\mathrm{SkP}_{T}$ and $\mathrm{SkO}_{T}$ [70], are able to reproduce empirical data involving s.p. levels in light nuclei.

This conclusion is nicely corroborated by results presented in Fig. 7, which shows the predictions for terminating states using the new Skyrme parameterization $\mathrm{SkP}_{L T}$. It is rewarding to see that the modified functional yields results that are very consistent with SM. This is particularly true for the isospin projection from the $\left|\left[d_{3 / 2}^{-1} f_{7 / 2}^{n+1}\right]_{I_{\text {max }}}^{\pi}\right\rangle$ configurations. The new values of $\Delta E$ reasonably agree with experiment, considering the energy range of the plot, While the detailed $A$-dependence is reproduced very well, theoretical $\Delta E$ curve slightly underestimates experiment. Identification of a specific source of this remaining discrepancy requires further studies.

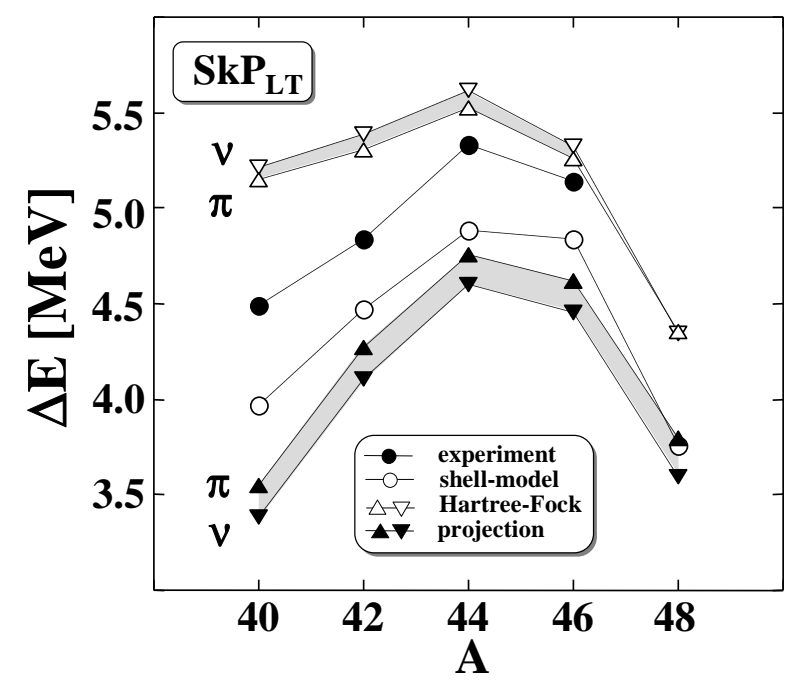

FIG. 7: Similar to Fig. [5 except for the modified Skyrme functional $\mathrm{SkP}_{L T}$ [59].

\section{SUMMARY AND CONCLUSIONS}

In this work, we carried out the theoretical analysis of isospin breaking in nuclei around $N=Z$ based on the density functional theory. We show that the spontaneous breaking of isospin symmetry inherent to MF limits the applicability of self-consistent theories, such as HF and DFT, to nuclear states with $T=0$. To remedy this problem, we propose a new isospin-symmetry restoration scheme based on the rediagonalization technique in goodisospin basis.

The isospin projection algorithm is described in detail, including the derivation of essential Hamiltonian kernels. We demonstrate that isospin projection is free from problems plaguing other symmetry restoration schemes (particle number, angular momentum) related to kernel singularities.

Applications of the isospin-projected DFT approach include the SD bands in ${ }^{56} \mathrm{Ni}$ and terminating states in $N=Z$ medium-mass nuclei. In both cases, the symmetry restoration remedies previously noted deficiencies of the HF method and significantly improves agreement with experiment. The results for terminating states corroborate previous suggestions that large isoscalar effective masses are needed to reproduce experimental spectroscopic data involving s.p. levels.

The examples presented in this work primarily concern high-spin configurations in medium mass nuclei in which pairing correlations are expected to be weak. To be able to address theoretically a variety of low-spin phenomena and observables around the $N=Z$ line, such as ground states of odd-odd nuclei, binding energy staggering, superallowed beta decays, and charge-exchange reactions, isovector and isoscalar pairing correlations must be incorporated and the proton-neutron symmetry of HF must be broken before isospin projection [46, 47]. Those will be 
subjects of our forthcoming studies.

This work was supported in part by the Polish Ministry of Science under Contracts No. N N202 328234 and N N202 239037, Academy of Finland and University of Jyväskylä within the FIDIPRO programme, and by the
Office of Nuclear Physics, U.S. Department of Energy under Contract Nos. DE-FG02-96ER40963 (University of Tennessee) and and DE-FC02-07ER41457 (UNEDF SciDAC Collaboration).
[1] P. Hohenberg and W. Kohn, Phys. Rev. 136, B864 (1964).

[2] W. Kohn and L.J. Sham, Phys. Rev. 140, A1133 (1965).

[3] M. Levy. Proc. Nat. Acad. Sci. 76, 6062 (1979).

[4] M. Levy. Phys. Rev. A 26, 1200 (1982).

[5] E. Lieb, Int. J. Quant. Chem. 24, 243 (1983).

[6] M. Levy and J. P. Perdew, in Density Functional Methods in Physics, edited by R.M. Dreizler and J. da Providencia (Plenum, New York, 1985).

[7] H. Englisch, H. Fieseler, and A. Haufe, Phys. Rev. A 37, 4570 (1988).

[8] A. Görling, Phys. Rev. A 54, 3912 (1996).

[9] M. Bender, P.-H. Heenen, and P.-G. Reinhard, Rev. Mod. Phys. 75, 121 (2003).

[10] Extended Density Functionals in Nuclear Structure Physics, ed. by G.A. Lalazissis, P. Ring, and D. Vretenar (Springer Verlag, 2004).

[11] E. Prodan, J. Phys. A 38, 5647 (2005).

[12] J. Engel, Phys. Rev. C 75, 014306 (2007).

[13] B.G. Giraud, Phys. Rev. C77, 014311 (2008).

[14] B.G. Giraud, B.K. Jennings, and B.R. Barrett, Phys. Rev. A78, 032507 (2008).

[15] J. Messud, M. Bender, and E. Suraud, Phys. Rev. C, in press; arXiv:0904.0162

[16] T.H.R. Skyrme, Phil. Mag. 1 (1956) 1043; Nucl. Phys. 9 (1959) 615.

[17] B.G. Carlsson, J. Dobaczewski, and M. Kortelainen, Phys. Rev. C 78, 044326 (2008).

[18] Isospin in nuclear physics, ed. D.H. Wilkinson (North Holland, Amsterdam, 1969).

[19] D.D. Warner, M.A. Bentley, and P. Van Isacker, Nature Physics 2, 311 (2006).

[20] D. Rudolph, C. Baktash, M.J. Brinkman, E. Caurier, D.J. Dean, M. Devlin, J. Dobaczewski, P.-H. Heenen, H.-Q. Jin, D.R. LaFosse, W. Nazarewicz, F. Nowacki, A. Poves, L.L. Riedinger, D.G. Sarantites, W. Satuła, and C.H. Yu, Phys. Rev. Lett. 82 (1999) 3763.

[21] M. Zalewski, W. Satuła, W. Nazarewicz, G. Stoitcheva, and H. Zduńczuk, Phys. Rev. C 75, 054306 (2007).

[22] J. Dobaczewski, M.V. Stoitsov, W. Nazarewicz, and P.G. Reinhard, C 76, 054315 (2007).

[23] D. Lacroix, T. Duguet, and M. Bender, Phys. Rev. C 79, 044318 (2009).

[24] T. Otsuka, M. Honma, and T. Mizusaki, Phys. Rev. Lett. 81 (1998) 1588.

[25] T. Mizusaki, T. Otsuka, M. Honma, and B.A. Brown, Phys. Scr. T88, 107 (2000).

[26] E. Caurier, A. Poves, and A. Zucker, Phys. Lett. 96B, 11 (1980); Phys. Lett. 96B, 15 (1980).

[27] E. Caurier and A. Poves, Nucl. Phys. A385, 407 (1982).

[28] M. Rafalski, W. Satuła, and J. Dobaczewski, Int. J. Mod. Phys. E18, 958 (2009); arXiv:0811.1135.

[29] W. Satuła, J. Dobaczewski, W. Nazarewicz, and M. Rafalski, Phys. Rev. Lett. 103, 012502 (2009).
[30] J.A. Sheikh and P. Ring, Nucl. Phys. A 665, 71 (2000).

[31] J.A. Sheikh, P. Ring, E. Lopes, and R. Rossignoli, Phys. Rev. C 66, 044318 (2002).

[32] M.V. Stoitsov, J. Dobaczewski, R. Kirchner, W. Nazarewicz, and J. Terasaki, Phys. Rev. C 76, 014308 (2007).

[33] G. Martinez-Pinedo, K. Langanke, and P. Vogel, Nucl. Phys. A 651, 379 (1999).

[34] P. Vogel, Nucl. Phys. A662, 148 (2000).

[35] J. Jänecke and T.W. O'Donnell, Phys. Lett. B 605, 87 (2005).

[36] C.A. Engelbrecht and R.H. Lemmer, Phys. Rev. Lett. 24, 607 (1970).

[37] D.A. Varshalovich, A.N. Moskalev, and V.K. Khersonskii, Quantum Theory of Angular Momentum (World Scientific, Singapore 1988).

[38] R. Rodriguez-Guzmán, J.L. Egido, and L.M. Robledo, Nucl. Phys. A709, 201 (2002).

[39] J.P. Blaizot and G. Ripka, Quantum theory of finite systems, MIT Press, Cambridge Mass., 1986.

[40] H. Zduńczuk, J. Dobaczewski, and W. Satuła, Int. J. Mod. Phys. E 16, 377 (2007).

[41] M. Beiner, H. Flocard, N. Van Giai, and P. Quentin, Nucl. Phys. A 238, 29 (1975).

[42] J. Dobaczewski, W. Nazarewicz, T.R. Werner, J.-F. Berger, C.R. Chinn, and J. Dechargé, Phys. Rev. C53, 2809 (1996).

[43] J. Dobaczewski, W. Satuła, B.G. Carlsson, J. Engel, P. Olbratowski, P. Powałowski, M. Sadziak, J. Sarich, N. Schunck, A. Staszczak, M.V. Stoitsov, M. Zalewski, and H. Zduńczuk, Comput. Phys. Commun. 180, 2361 (2009).

[44] W.M. MacDonald, in Isobaric Spin in Nuclear Physics, Ed. by J.D. Fox and D. Robson, Academic Press, 1966.

[45] A.Z. Mekjian and W.M. MacDonald, Nucl. Phys. A 121, 385 (1968).

[46] E. Perlińska, S.G. Rohoziński, J. Dobaczewski, and W. Nazarewicz, Phys. Rev. C 69, 014316 (2004).

[47] S.G. Rohoziński, J. Dobaczewski, and W. Nazarewicz, arXiv:0910.5270

[48] M. Anguiano, J.L. Egido, and L.M. Robledo, Nucl. Phys. A 696, 467 (2001).

[49] L.M. Robledo, Int. J. Mod. Phys. E 16, 337 (2007).

[50] J.C. Hardy and I.S. Towner, Phys. Rev. C71, 055501 (2005); Phys. Rev. Lett. 94, 092502 (2005); Phys. Rev. C79, 055502 (2009).

[51] I.S. Towner and J.C. Hardy, Phys. Rev. C 77, 025501 (2008).

[52] G.A. Miller and A. Schwenk, Phys. Rev. C 78, 035501 (2008).

[53] L.A. Sliv and Yu.I. Kharitonov, Phys. Lett. 16, 176 (1965).

[54] A. Bohr, J. Damgård, and B. Mottelson, in Nuclear Structure, ed. by A. Hossian et al. (North-Holland Publ. 
Co., Amsterdam, 1967)

[55] E. Chabanat, P. Bonche, P. Haensel, J. Meyer, and R. Schaeffer, Nucl. Phys. A627 (1997) 710.

[56] J. Dobaczewski, H. Flocard and J. Treiner, Nucl. Phys. A422, 103 (1984).

[57] J. Dobaczewski et al., Comput. Phys. Commun. 102, 166 (1997); 102, 183 (1997); 131, 164 (2000); 158, 158 (2004); 167, 214 (2005); 180, 2361 (2009).

[58] J. Dobaczewski, B.G. Carlsson, J. Dudek, J. Engel, P. Olbratowski, P. Powałowski, M. Sadziak, J. Sarich, W. Satuła, N. Schunck, A. Staszczak, M.V. Stoitsov, M. Zalewski, and H. Zduńczuk, HFODD (v2.40h): User's Guide, arXiv:0909.3626

[59] M. Zalewski, J. Dobaczewski, W. Satuła, and T.R. Werner, Phys. Rev. C 77, 024316 (2008).

[60] S.M. Lenzi, D.R. Napoli, C.A. Ur, D. Bazzacco, F. Brandolini, J.A. Cameron, E. Caurier, G. de Angelis, M. De Poli, E. Farnea, A. Gadea, S. Hankonen, S. Lunardi, G. Martínez-Pinedo, Zs. Podolyak, A. Poves, C. Rossi Alvarez, J. Sánchez-Solano, and H. Somacal, Phys Rev. C60, 021303(R) (1999).

[61] P. Bednarczyk, W. Męczyński, J. Styczeń, J. Grȩbosz, M. Lach, A. Maj, M. Ziębliński, N. Kintz, J.C. Merdinger, N. Schulz, J.P. Vivien, A. Bracco, J.L. Pedroza, M.B. Smith, and K.M. Spohr, Acta Phys. Pol. B32, 747 (2001).

[62] F. Brandolini, N.H. Medina, D. Bazzacco, J.A. Cameron, G. de Angelis, A. Gadea, N. Menegazzo, and C. RossiAlvarez, Nucl. Phys. A693, 517 (2001).

[63] M. Lach, J. Styczeń, W. Męczyński, P. Bednarczyk, A. Bracco, J. Grȩbosz, A. Maj, J.C. Merdinger, N. Schultz, M.B. Smith, K.M. Spohr, J.P. Vivien, and M. Ziębiński, Eur. Phys. J. A16, 309 (2003).

[64] M. Lach, J. Styczeń, W. Mȩczyński, P. Bednarczyk, A. Bracco, J. Grȩbosz, A. Maj, J.C. Merdinger, N. Schultz, M.B. Smith, K.M. Spohr, and M. Ziębiński, Eur. Phys. J. A25, 1 (2005).
[65] C.J. Chiara, M. Devlin, E. Ideguchi, D.R. LaFosse, F. Lerma, W. Reviol, S.K. Ryu, D.G. Sarantites, O.L. Pechenaya, C. Baktash, A. Galindo-Uribarri, M.P. Carpenter, R.V.F. Janssens, T. Lauritsen, C.J. Lister, P. Reiter, D. Seweryniak, P. Fallon, A. Gorgen, A.O. Macchiavelli, D. Rudolph, G. Stoitcheva, and W.E. Ormand, Phys. Rev. C75, 054305 (2007); C75, 059904(E) (2007).

[66] H. Zduńczuk, W. Satuła, J. Dobaczewski, and M. Kosmulski, Phys. Rev. C76, 044304 (2008).

[67] H. Zduńczuk, W. Satuła, and R. Wyss, Phys. Rev. C71 (2005) 024305; Int. J. Mod. Phys. E14 (2005) 451; W. Satuła, R. Wyss, and H. Zduńczuk, Eur. Phys. J. A 25, s01, (2005) 551. .

[68] G. Stoitcheva, W. Satuła, W. Nazarewicz, D.J. Dean, M. Zalewski and H. Zduńczuk, Phys. Rev. C73, 061304 (2006).

[69] M. Zalewski, W. Satuła, W. Nazarewicz, G. Stoitcheva, and H. Zduńczuk, Phys. Rev. C75, 054306 (2007).

[70] W. Satuła, R.A. Wyss and M. Zalewski, Phys. Rev. 78, 011302(R) (2008).

[71] W. Satuła, Int. J. Mod. Phys. E16, 360 (2007).

[72] T. Lesinski, M. Bender, K. Bennaceur, T. Duguet, and J. Meyer, Phys. Rev. C 76, 014312 (2007).

[73] J. Toivanen, J. Dobaczewski, M. Kortelainen, and K. Mizuyama, Phys. Rev. C 78, 034306 (2008).

[74] P. Klüpfel, P.-G. Reinhard, T.J. Burvenich, and J.A. Maruhn, Phys. Rev. C79, 034310 (2009).

[75] M. Bender, K. Bennaceur, T. Duguet, P.-H. Heenen, T. Lesinski, and J. Meyer, Phys. Rev. C, in press; arXiv:0909.3782

[76] M. Kortelainen, J. Dobaczewski, K. Mizuyama, and J. Toivanen, Phys. Rev. C 77, 064307 (2008).

[77] W. Satuła, J. Dobaczewski, W. Nazarewicz, and M. Rafalski, to be published. 